\title{
The role of FDG PET/CT in detection of distant metastasis in the initial staging of breast cancer
}

\author{
Anar ALIYEV ${ }^{1}$, Sabire YILMAZ AKSOY ${ }^{1}$, Meftune ÖZHAN ${ }^{1}$, Özgül EKMEKÇİOĞLU ${ }^{1}$, Betül VATANKULU ${ }^{1}$, \\ Pınar Çiğdem KOCAEL ${ }^{2}$, Muhammet Sait SAĞER ${ }^{1}$, Melih Engin ERKAN ${ }^{3}$, Kerim SÖNMEZOĞLU ${ }^{1}$, Metin HALAÇ $^{1{ }^{*}}$ \\ ${ }^{2}$ Department of Nuclear Medicine, Cerrahpaşa Faculty of Medicine, İstanbul University, İstanbul, Turkey \\ ${ }^{2}$ Department of General Surgery, Cerrahpaşa Faculty of Medicine, İstanbul University, İstanbul, Turkey \\ ${ }^{3}$ Department of Nuclear Medicine, Faculty of Medicine, Düzce University, Düzce, Turkey
}

Background/aim: We aimed to evaluate the role of FDG PET/CT in the detection of extraaxillary regional nodal/distant metastasis in breast cancer patients and to assess the value of FDG PET/CT for detecting distant metastases in patient subgroups.

Materials and methods: A total of 254 patients with breast cancer (248 female, 6 male) who underwent PET/CT for initial staging were enrolled. Patients were divided into four groups: Group 1 consisted of 154 patients diagnosed by tru-cut/core/FNAB, Group 2 comprised 32 patients diagnosed by excisional biopsy, Group 3 included 62 patients who had mastectomy-axillary lymph node dissection, and Group 4 consisted of 6 patients who had axillary lymph node metastasis diagnosed by excisional biopsy.

Results: PET/CT detected distant metastasis in 76 of the 254 patients. Of these patients, $21.7 \%$ had bone/bone marrow metastasis, $7.1 \%$ had lung metastasis, $13 \%$ had mediastinal lymph node metastasis, $4.8 \%$ had liver metastasis, 9.8\% had other organ/system metastasis, and $6 \%$ had other lymphadenopathies. According to T staging, the percentages of distant metastasis were as follows: $13.6 \%$ of the $66 \mathrm{~T} 1$ stage patients, $35.7 \%$ of the $129 \mathrm{~T} 2$ stage patients, $40 \%$ of the $20 \mathrm{~T} 3$ stage patients, and $33.3 \%$ of the 39 T 4 stage patients.

Conclusion: FDG-PET/CT led to a change in the stage of disease and the treatment approach in newly diagnosed breast cancer patients due to its superiority in detecting extraaxillary regional lymph node metastases and distant metastases.

Key words: FDG PET/CT, breast cancer, distant metastasis

\section{Introduction}

Breast cancer is the most common type of cancer in women and the second leading cause of death by cancer among women (1). The 5 -year survival rate is about $80 \%$ in local breast cancer, whereas it is $25 \%$ in metastatic breast cancer; the presence of distant metastases is the most important prognostic factor in breast cancer (2). Hence, accurate initial staging is the most important indicator in choosing a treatment modality in breast cancer. The risk of developing breast cancer $(0-110$ age) is $7 \%-10 \%$ in women during their lifetime and approximately one in every 10 to 14 women develops breast cancer. Male breast carcinoma constitutes about $1 \%$ of all breast cancers (3). Breast cancer is a rare disease under the age of 25 . The risk of breast cancer increases as a woman gets older. Most breast cancers occur between the ages of 45 and 75 years (4).

The choice of a treatment modality in early invasive breast cancer involves a multidisciplinary approach including the combination of surgery, radiotherapy (RT),

\footnotetext{
*Correspondence: metin.halac@yahoo.com
}

and chemotherapy. The main goal of surgical treatment is the optimal control of tumor in the breast and axilla. Systemic chemotherapy and hormonotherapy aim to treat possible undetermined micrometastases. The treatment modalities for the disease when it is limited to the breast and the regional lymph nodes are usually surgery, adjuvant chemotherapy, and hormonotherapy. For metastatic cases, however, less aggressive palliative treatments are preferred. The primary treatment is chemotherapy, and in some instances RT in cases of distant metastatic disease. Such patients rarely require surgical treatment (simple mastectomy, metastasectomy, etc.).

Ultrasonography (US), mammography, and magnetic resonance imaging (MRI) are the standard imaging techniques in the diagnosis of breast cancer. However, these modalities provide anatomical information rather than information on metabolic activity, which is correlated with prognosis. Additionally, these methods cannot show extraaxillary regional lymph node metastases and distant 
metastases. Locally advanced breast cancer has a poor prognosis because of its high rate of distant metastases (5). The National Comprehensive Cancer Network recommends the use of chest radiography (CXR), mammography, and breast US as necessary, and optional additional studies as indicated by symptoms such as breast MRI, skeletal scintigraphy, computed tomography (CT), US, or MRI of the abdomen; CT, US, or MRI of the pelvis; and ${ }^{18} \mathrm{~F}$-fluorodeoxyglucose (FDG) positron emission tomography (PET)/CT scan for staging of locally advanced breast cancer at the time of diagnosis. CT and MRI can be helpful for clarification of suspected cases (6). Despite negative findings with conventional imaging methods, some patients present with distant metastases a short time later. Thus, the role of aggressive local treatment is controversial in this group of patients. More advanced imaging methods are needed for the detection of possible distant metastases in order to prevent ineffective aggressive local treatment. Early detection of distant metastases and their proper management provide both better prognosis and a reduction in unnecessary procedures.

Malignant tumors have increased glucose metabolism and PET/CT using FDG can detect these lesions effectively (7). FDG PET/CT, which provides both anatomical and functional information, allows diagnosis, staging, evaluation of response to therapy, and detection of recurrences in many types of cancer $(7,8)$. FDG PET has been used for the detection of primary tumors, lymph node metastases, and distant metastases in breast cancer and it is superior to conventional imaging methods in the detection of distant metastases (9-11). FDG PET has a sensitivity, specificity, and accuracy of $87 \%, 83 \%$, and $87 \%$, respectively, in detecting distant metastases in breast cancer. Combined conventional imaging methods include CXR, US of the abdomen and the axilla, and bone scintigraphy and plain-film X-rays of the bones, if necessary, which have a sensitivity and specificity of $43 \%$ and $98 \%$, respectively. Sensitivity, specificity, and accuracy of CT in detecting distant metastases in breast cancer were reported to be $83 \%, 85 \%$, and $84 \%$, respectively (11).

There is a limited number of studies concerning the role of FDG PET/CT in the staging of breast cancer when compared with those about conventional imaging methods (including CT). It was suggested that PET/CT is an effective imaging modality in detecting occult metastases in breast cancer. One study with a limited number of patients showed that PET/CT can determine occult metastases in $10 \%$ of patients with stage $2-3$ breast cancer (12). In a recent study examining the detection of distant metastases in breast cancer by PET/CT, the sensitivity and specificity were $97.4 \%$ and $91.2 \%$, respectively, while by the conventional imaging methods they were $85.9 \%$ and $67.3 \%$, respectively (13). Although the role of PET/CT in the initial staging of breast cancer and the routine usage of $\mathrm{PET} / \mathrm{CT}$ is controversial, we thought that PET/CT can be cost-effective on the basis of these data.

Here, we performed a retrospective study to identify the role of FDG PET/CT in detecting extraaxillary regional lymph nodes and distant metastases in patients with breast cancer who were referred to our department for FDG PET/CT.

\section{Materials and methods}

\subsection{Patient group}

Between May 2005 and June 2011, a total of 254 patients ( 248 women, 6 men, mean age: $54.3 \pm 13.8$ ) with breast cancer who underwent PET/CT for initial staging were included in this study. These patients were composed of four separate groups: 154 patients with breast cancer diagnosed by tru-cut/core or FNAB (Group 1), 32 patients diagnosed by excisional biopsy (Group 2), 62 patients who had mastectomy and axillary lymph node dissection (Group 3), and 6 patients who had axillary lymph node metastasis diagnosed by excisional biopsy (Group 4). Of these patients, 203 had invasive ductal carcinoma, 13 had invasive lobular carcinoma, 6 had invasive mucinous cancers, 15 had invasive ductal/lobular cancers, and 17 had other than these histopathological results. A total of 129 patients had right breast cancer, 119 had left breast cancer, and 6 patients had bilateral breast cancer. In terms of disease stage, 66 had T1 stage disease, 129 had T2 stage disease, 20 had T3 stage disease, and 39 had T4 stage disease (Table 1).

Table 1. Patient groups and T stages.

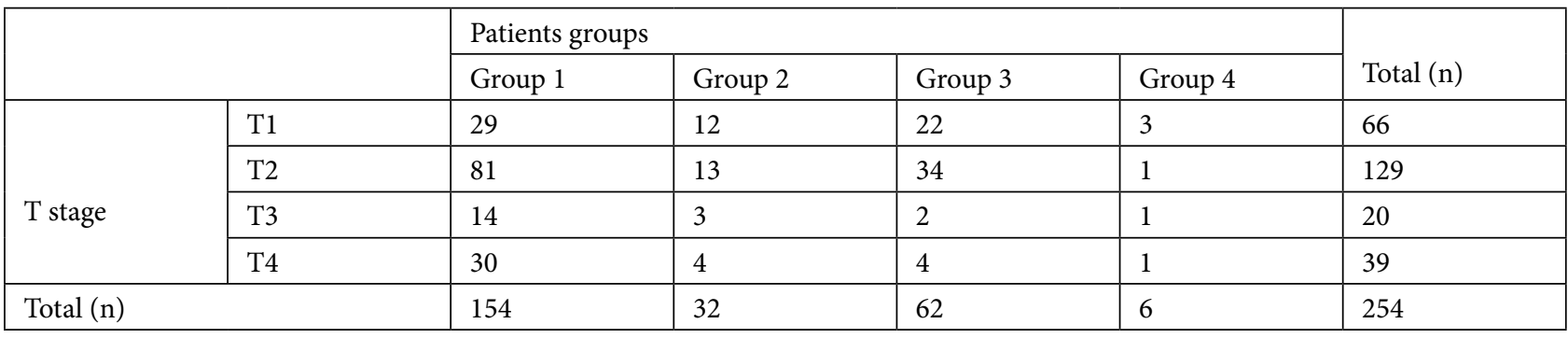


Of the 6 male patients (mean age: $63.3 \pm 14.0$ ), 5 patients were diagnosed by tru-cut/core or fine needle aspiration (FNA) biopsy and 1 patient was diagnosed by mastectomy and axillary dissection. All 6 patients had invasive ductal carcinoma; 2 of them were in the right breast and 4 were in the left breast. One of the 6 patients was stage T1, 3 were $\mathrm{T} 2$, and 2 were $\mathrm{T} 4$.

In Group 1, of the 154 patients with breast cancer diagnosed by tru-cut or FNA biopsy, 127 patients had invasive ductal cancer, 7 had invasive lobular cancer, 5 had invasive mucinous cancer, 7 had invasive lobular/ ductal cancer, 1 had bilateral breast cancer with different pathologies, and 7 had other than these pathologic results. In 74 patients the cancer was in the right breast, in 76 it was in the left breast, and in 4 it was bilateral breast cancer. Twenty-nine of the 154 patients were stage $\mathrm{T} 1,81$ were $\mathrm{T} 2$, 14 were $\mathrm{T} 3$, and 30 were $\mathrm{T} 4$ (Table 1 ).

In Group 2, of the 32 patients with breast cancer diagnosed by excisional biopsy, 23 patients had invasive ductal cancer, 2 had invasive lobular cancer, 1 had invasive mucinous cancer, 1 had invasive lobular/ductal cancer, and 5 had pathologic results other than these. Of the 32 patients, 17 had right breast cancer and 15 had left breast cancer. Twelve of the 32 patients were stage T1, 13 were T2, 3 were T3, and 4 were T4 (Table 1 ).

In Group 3, of the 62 patients with breast cancer who had mastectomy and axillary dissection, 49 patients had invasive ductal cancer, 3 had invasive lobular cancer, 7 had invasive lobular/ductal cancer, 1 had bilateral breast cancer with different pathologies, and 2 had pathologic results other than these. Of the 62 patients, 35 had rightsided breast cancer, 25 had left-sided breast cancer, and 2 had bilateral breast cancer. Twenty-two of the 62 patients were stage T1, 34 were T2, 2 were T3, and 4 were T4 (Table $1)$.

In Group 4, of the 6 patients with breast cancer diagnosed by excisional biopsy of axillary lymphadenopathy, 4 patients had invasive ductal cancer, 1 had invasive lobular cancer, and 1 had pathologic results other than these. Three of the 6 patients had right breast cancer and the other 3 had left breast cancer. Three of 6 patients were stage T1, 1 was T2, 1 was T3, and 1 was T4 (Table 1).

\subsection{PET/CT imaging and review}

PET/CT images were acquired from the skull base to midthighs 1-1.5 h postinjection of FDG using a dedicated full-ring LSO based PET scanner (Siemens Biograph LSO HI-REZ PET/BT, USA) integrated with a 6-slice CT. Patients fasted for at least $4 \mathrm{~h}$ before the FDG injections. No intravenous contrast was given for the CT part of the study. The images were corrected for attenuation. Attenuation corrected PET images were interpreted first, and nonattenuation corrected images were also examined when needed. FDG uptake that was greater than the background physiological activity was considered to be significant.

On FDG PET/CT imaging, FDG-positive lesions were categorized as extraaxillary regional lymph nodes metastases (ipsilateral suprainfraclavicular-internal mammary lymphadenopathies) and distant metastases. Distant metastases were classified as contralateral axillarysupraclavicular-internal mammary lymphadenopathies, bone lesions, lung lesions, mediastinal lymphadenopathies (LAPs), liver lesions, and other lesions. Furthermore, the interpretation of lesions was based primarily on visual assessment of the intensity of FDG uptake, calculation of the SUVmax, and evaluation of CT appearances.

Verification of the FDG-PET/CT findings was performed with histopathological examination, radiological correlation, and clinical and PET imaging follow-ups.

\subsection{Data analysis}

Statistical analysis was performed using SPSS 12 (ver. 12.0 for Windows; SPSS Inc., USA). Data are presented as means \pm standard deviation (SD) and minimum-maximum values; independent samples t-test was applied to examine the differences between the groups. A chi-squared test was used to calculate the differences in qualitative categorical data between groups, and the results were expressed as frequencies (\%). P $<0.05$ was considered significant.

\section{Results}

Among the 254 patients with breast cancer who underwent FDG PET/CT for initial staging, 44 (17.3\%) had ipsilateral suprainfraclavicular lymph node metastases and 32 (12.6\%) had ipsilateral parasternal lymph node metastases. Additionally, in 1 patient, a false-positive increase in FDG uptake that was initially suggested to be ipsilateral supraclavicular lymph node metastases was observed. Histopathological examination confirmed inflammatory changes.

Distant metastases were seen in 76 (29.9\%) of the 254 patients with breast cancer who underwent FDG PET/ CT for initial staging. Of these 76 patients, 55 patients (21.7\%) had bone/bone marrow metastasis (17 solitary, 38 multiple), 18 patients (7.1\%) had lung metastasis ( 4 solitary, 14 multiple), 33 patients (13\%) had mediastinal lymph node metastasis, 12 patients $(4.8 \%)$ had liver metastasis (4 solitary, 13 multiple), 25 patients $(9.8 \%)$ had other organ/system metastasis (12 solitary, 13 multiple), and 15 patients $(6 \%)$ had contralateral axillary-supraclavicularinternal mammary lymphadenopathies (5 contralateral axillary, 5 contralateral supraclavicular, and 5 contralateral axillary-supraclavicular).

According to the $\mathrm{T}$ stage, the rate of distant metastases was as follows: $9(13.6 \%)$ of the $66 \mathrm{~T} 1$ stage patients, 46 (35.7\%) of the $129 \mathrm{~T} 2$ stage patients, $8(40 \%)$ of the $20 \mathrm{~T} 3$ 
stage patients, and 13 (33.3\%) of the $39 \mathrm{~T} 4$ stage (Table 2). It is noteworthy that the rate of detected distant metastases increased as the $\mathrm{T}$ stage increased until the $\mathrm{T} 3$ stage $(\mathrm{P}=$ 0.015).

Distant metastases were found in 64 (41.6\%) of the 154 patients with known breast cancer diagnosed by tru-cut/ core or FNA biopsy (Group 1) who underwent FDG-PET/ CT for initial staging. Of these 64 patients, 46 (29.9\%) had bone/bone marrow metastasis (12 solitary, 34 multiple), 17 patients had lung metastasis (4 solitary, 13 multiple), 29 (18.8\%) patients had mediastinal lymph node metastasis, 9 (5.8\%) patients had liver metastasis (2 solitary, 7 multiple), 21 patients $(13.6 \%)$ had other organ/system metastasis (10 solitary, 11 multiple), and 14 patients (9\%) had contralateral axillary-supraclavicular-internal mammary lymphadenopathies (5 with contralateral axillary, 4 with contralateral supraclavicular, and 5 with contralateral axillary-supraclavicular lymphadenopathies) (Figures 1 and 2).

The rate of distant metastasis according to $\mathrm{T}$ stage in Group 1 patients was as follows: $8(27.5 \%)$ of the $29 \mathrm{~T} 1$ stage patients, $37(45.6 \%)$ of the $81 \mathrm{~T} 2$ stage patients, $7(50 \%)$ of the 14 T3 stage patients, and $12(40 \%)$ of the 30 T4 stage patients (Table 3). Although not significant $(\mathrm{P}=0.471)$, it was observed that the frequency of distant metastases increased as the stage increased until the T3 stage.

In Group 1, the mean SUVmax value of the primary lesion in 64 patients with distant metastases on FDG PET/ CT was $10.9 \pm 5.6(2.1-26.8)$, while the mean SUVmax value of the primary lesion in 90 patients with no distant metastases was $8.7 \pm 5.6(2.5-25.2)$; the difference was significant $(P=0.022$, Figure 3$)$.

Distant metastases were found in $8(25 \%)$ of the 32 patients with breast cancer diagnosed by excisional biopsy
(Group 2). Of these 8 patients, 5 (15.6\%) had bone/bone marrow metastasis (4 solitary, 1 multiple), 3 (9.4\%) had mediastinal lymph node metastasis, 1 (3.1\%) had liver metastasis (solitary), and 3 (9.3\%) had other organ/system metastasis (1 solitary, 2 multiple). The rate of distant metastasis according to $\mathrm{T}$ stage in Group 2 patients is as follows: $1(8.3 \%)$ of the $12 \mathrm{~T} 1$ stage patients, $5(38.5 \%)$ of the $13 \mathrm{~T} 2$ stage patients, $1(33.3 \%)$ of the $3 \mathrm{~T} 3$ stage patients, and $1(25 \%)$ of the $4 \mathrm{~T} 4$ stage patients.

Distant metastases were found in 4 of 62 patients (6.5\%) with breast cancer who had mastectomy and axillary dissection (Group 3). Of these 4 patients, 4 (6.5\%) had bone/bone marrow metastasis (1 solitary, 3 multiple), 1 patient (1.6\%) had lung metastasis (multiple), 1 patient (1.6\%) had mediastinal lymph node metastasis, 2 patients (3.2\%) had liver metastasis (1 solitary, 1 multiple), 1 patient (1.6\%) had other organ/system metastasis (solitary), and 1 patient (1.6\%) had contralateral supraclavicular lymph node metastasis (Figure 4). When we look at the presence of distant metastases according to $\mathrm{T}$ stage in Group 3 patients, distant metastases were seen only in 4 of the 22 patients with $\mathrm{T} 2$ stage.

Of the 6 patients in Group 4 who were diagnosed with breast cancer by excisional biopsy of axillary LAP via histopathological examination, 3 were T1 stage, 1 was T2 stage, 1 was T3 stage, and 1 was T4 stage. Variable degrees of increased FDG uptake (SUDmax: $5.3 \pm 32.1$ ) were observed in the primary lesions of all 6 patients. None of the patients in this group had extraaxillary regional lymph nodes or distant metastases.

Of the 6 male patients (mean age $=63.3 \pm 14.0$ ), 5 patients were diagnosed by tru-cut/core or fine needle aspiration (FNA) biopsy and 1 patient with known breast cancer underwent mastectomy and axillary dissection. While all 6 patients had invasive ductal carcinoma

Table 2. The frequency of distant metastases with respect to T stages in 254 patients who underwent FDG PET/CT for initial staging.

\begin{tabular}{|l|l|l|l|l|l|}
\hline \multicolumn{2}{|c|}{} & \multicolumn{2}{l|}{ Primary staging } & \multirow{2}{*}{ Total } \\
\cline { 3 - 5 } \multicolumn{2}{|c|}{} & Metastases (-) & Metastases (+) & \\
\hline & I & n & 57 & 9 & 66 \\
\hline & & $\%$ & 86.4 & 13.6 & 100.0 \\
\hline & II & n & 83 & 46 & 129 \\
\hline & & $\%$ & 64.3 & 35.7 & 100.0 \\
\hline T stage & III & n & 12 & 8 & 20 \\
\hline & & $\%$ & 60.0 & 40.0 & 100.0 \\
\hline & IV & n & 26 & 13 & 39 \\
\hline & & $\%$ & 66.7 & 33.3 & 100.0 \\
\hline Total & n & 177 & 77 & 254 \\
\hline & & $\%$ & 69.7 & 30.3 & 100.0 \\
\hline
\end{tabular}




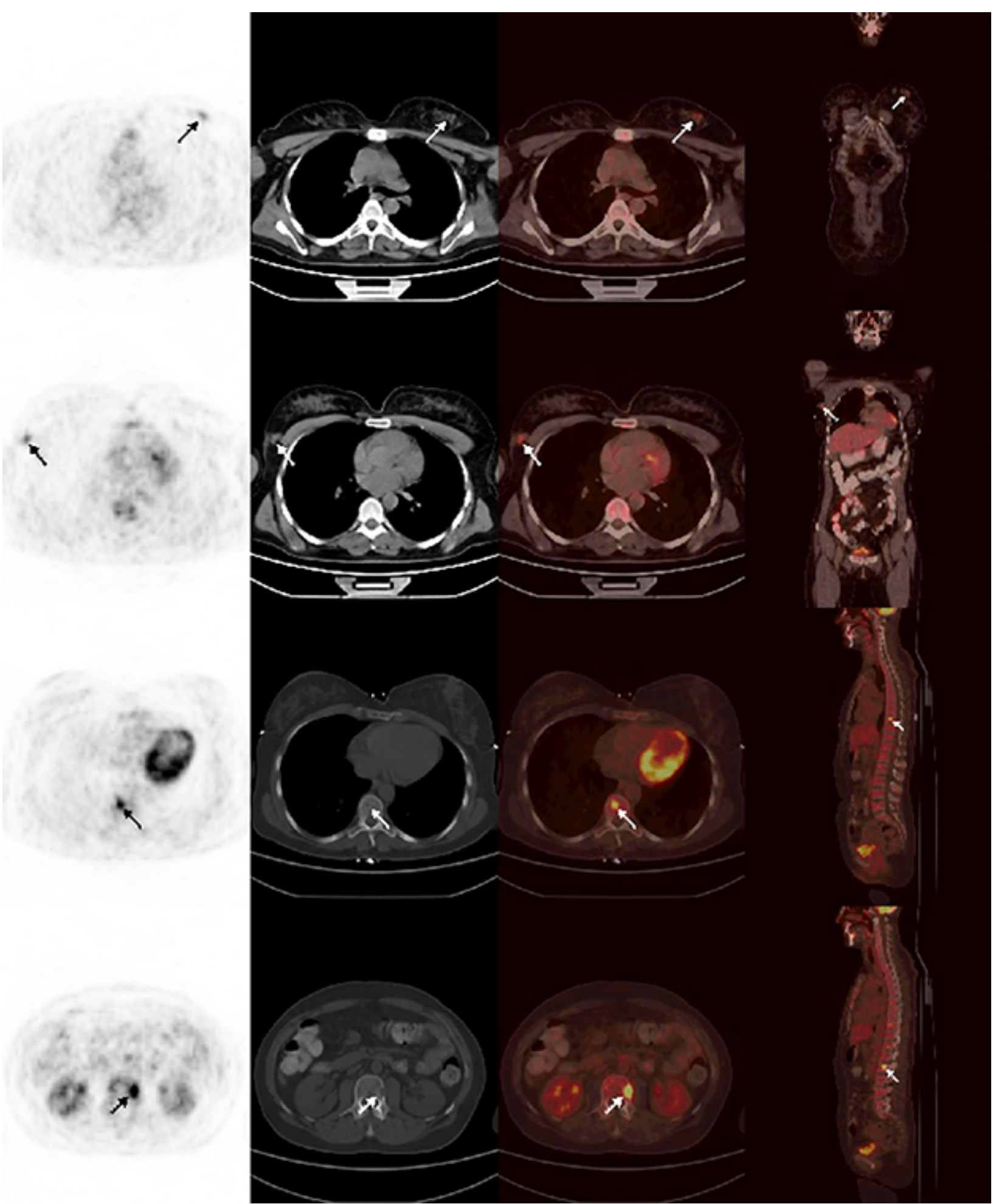

Figure 1. PET/CT images of a 51-year-old female patient. The biopsy findings from both breast lesions revealed invasive ductal carcinoma. The PET/CT performed for the purpose of staging showed hypermetabolism in the known malignant tumoral lesions localized in the upper central part of the left breast (1st row) and the upper outer quadrant of the right breast (2nd row). In this case without pathological findings in both axillae, hypermetabolic lesions suggesting metastases were detected in the right side of the D9 vertebral body (3rd row) and the left side of the L2 vertebral body. 


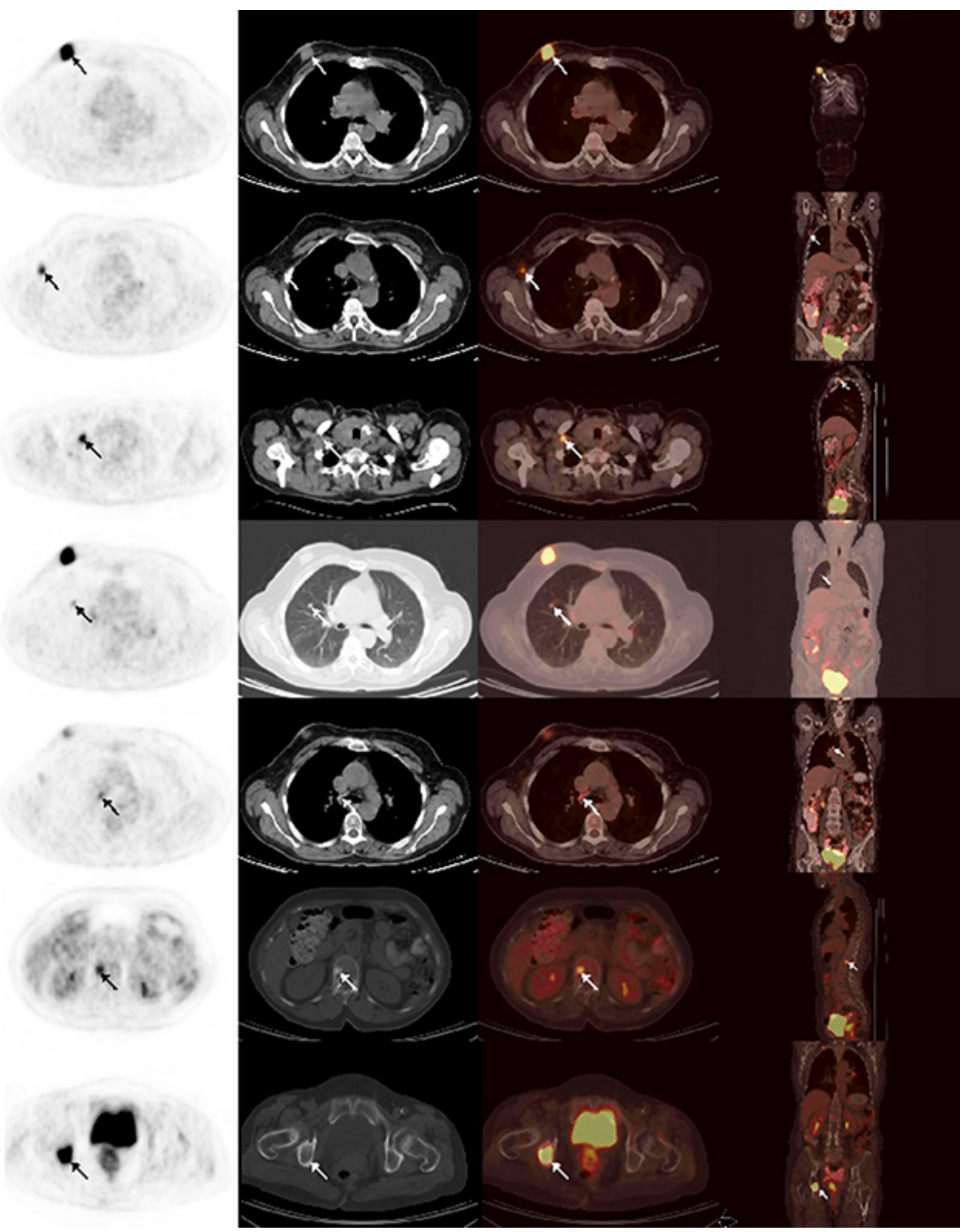

Figure 2. A 71-year-old female patient underwent PET/CT for initial staging. The biopsy findings from the right breast lesion revealed invasive ductal carcinoma. Intense hypermetabolism was observed in known malignant tumoral lesion localized in the upper inner quadrant of right breast (1st row). Furthermore, hypermetabolic lymph nodes in the right axilla (2nd row) and right retroclavicular area (3rd row) consistent with metastases were seen on the PET/CT images. In addition, millimeter-sized multiple nodular lesions with mild-minimal hypermetabolism were observed in both lungs (4th row), suggesting metastases. In the right lower paratracheal mediastinal and bilateral hilar LAPs (5th row) mild hypermetabolism was also seen, suggesting metastases. In the skeletal system, there were metastatic hypermetabolic lesions in the right side of the L1 vertebral body and right ischium. 
Table 3. The frequency of distant metastases with respect to T stages in 154 patients of Group 1.

\begin{tabular}{|c|c|c|c|c|c|}
\hline & & & \multicolumn{2}{|c|}{ Primary staging } & \multirow{2}{*}{ Total } \\
\hline & & & Metastases - & Metastases + & \\
\hline & I & $\mathrm{N}$ & 21 & 8 & 29 \\
\hline & & $\%$ & 72.5 & 27.5 & 100.0 \\
\hline & II & $\mathrm{N}$ & 44 & 37 & 81 \\
\hline & & $\%$ & 54.4 & 45.6 & 100.0 \\
\hline \multirow[t]{4}{*}{ T stage } & III & $\mathrm{N}$ & 7 & 7 & 14 \\
\hline & & $\%$ & 50.0 & 50.0 & 100.0 \\
\hline & IV & $\mathrm{N}$ & 18 & 12 & 30 \\
\hline & & $\%$ & 60.0 & 40.0 & 100.0 \\
\hline \multirow{2}{*}{\multicolumn{2}{|c|}{ Total }} & $\mathrm{N}$ & 90 & 64 & 154 \\
\hline & & $\%$ & 58.5 & 41.5 & 100.0 \\
\hline
\end{tabular}

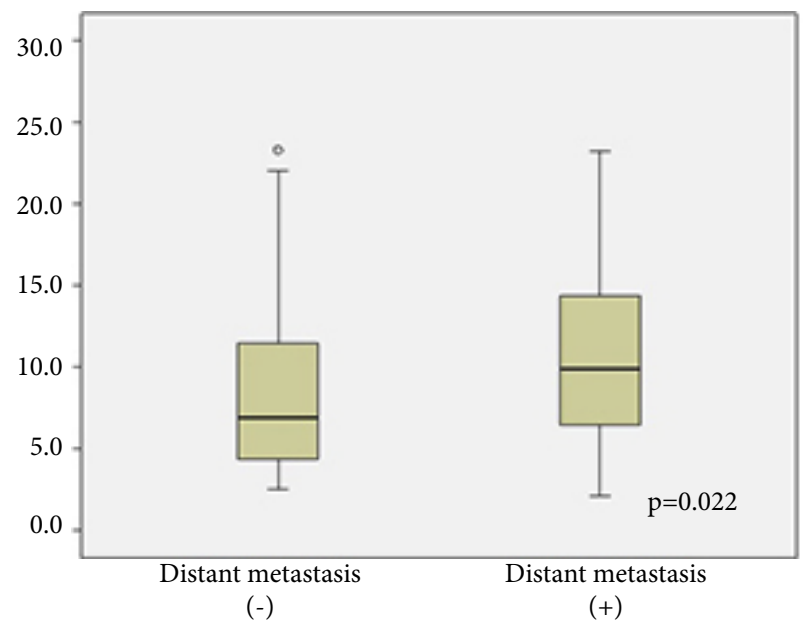

Figure 3. Comparison of SUVmax values of primary lesions in patients with/without distant metastases in Group 1.

diagnosis, 2 of them had right breast cancer and 4 had left breast cancer. One of the 6 patients was stage T1, 3 were $\mathrm{T} 2$, and 2 were $\mathrm{T} 4$.

One patient was diagnosed via a left breast mass excision, although there was no pathological FDG uptake in regional lymph node stations and visceral organsskeletal systems; second primary disease was suspected due to the multiple LAPs with increased FDG uptake in the abdomen. Histopathological examination of the abdominal LAPs confirmed the diagnosis of follicular lymphoma. In another patient with breast cancer diagnosed by biopsy from the right breast, there was increased FDG uptake at the primary lesion in the right breast and right axillary lymph nodes, which was consistent with metastases and tumoral lesion with very intense FDG accumulation at the sigmoid colon, which was considered for second primary. In this case, high-grade tubulovillous adenoma was diagnosed through a histopathological examination.

There were false-positive foci with increased FDG uptake in 2 patients. One of them had bilateral mediastinal lymphadenopathies with intense FDG uptake, and the other had a nodular lesion with increased FDG uptake at the apical segment of the upper lobe of the right lung and a mass lesion with intense FDG accumulation at the left inguinal fossa. These lesions were histopathologically confirmed as inflammatory lesions. Additionally, in 1 patient with right breast cancer, bilateral multiple millimetric lung nodules on CT images with no FDG uptake because of resolution limit and/or FDG affinity were considered false-negative. In this case there was a right internal mammary lymph node with increased FDG uptake, which was suspected to be metastatic.

\section{Discussion}

FDG-PET has proven to be an effective imaging modality for detecting distant metastases in the initial staging of breast cancer $(11,14-16)$. Schirrmeister et al. (14) studied 93 patients and they found that $12(12.9 \%)$ patients had internal mammary lymph node metastasis and 6 (6.5\%) patients had distant metastases detected by FDG PET. In another study, 4 of 84 (5\%) patients had distant metastases found on FDG-PET and 1 patient had a second primary lung tumor. In a study by Cermik et al. (15) including 240 patients with breast cancer who underwent FDG-PET for initial staging, extraaxillary lymph nodes/distant organ metastases were found in $22(9.2 \%)$ of these patients, whereas solitary multiple distant metastases were detected in 18 (7.5\%) of them, which caused changes in the TNM stage of the disease by FDG PET. In a study by Mahner et al. (11) distant metastases were detected in 18 (26\%) of 69 patients (11 bone and bone marrow, 7 lung, and 4 


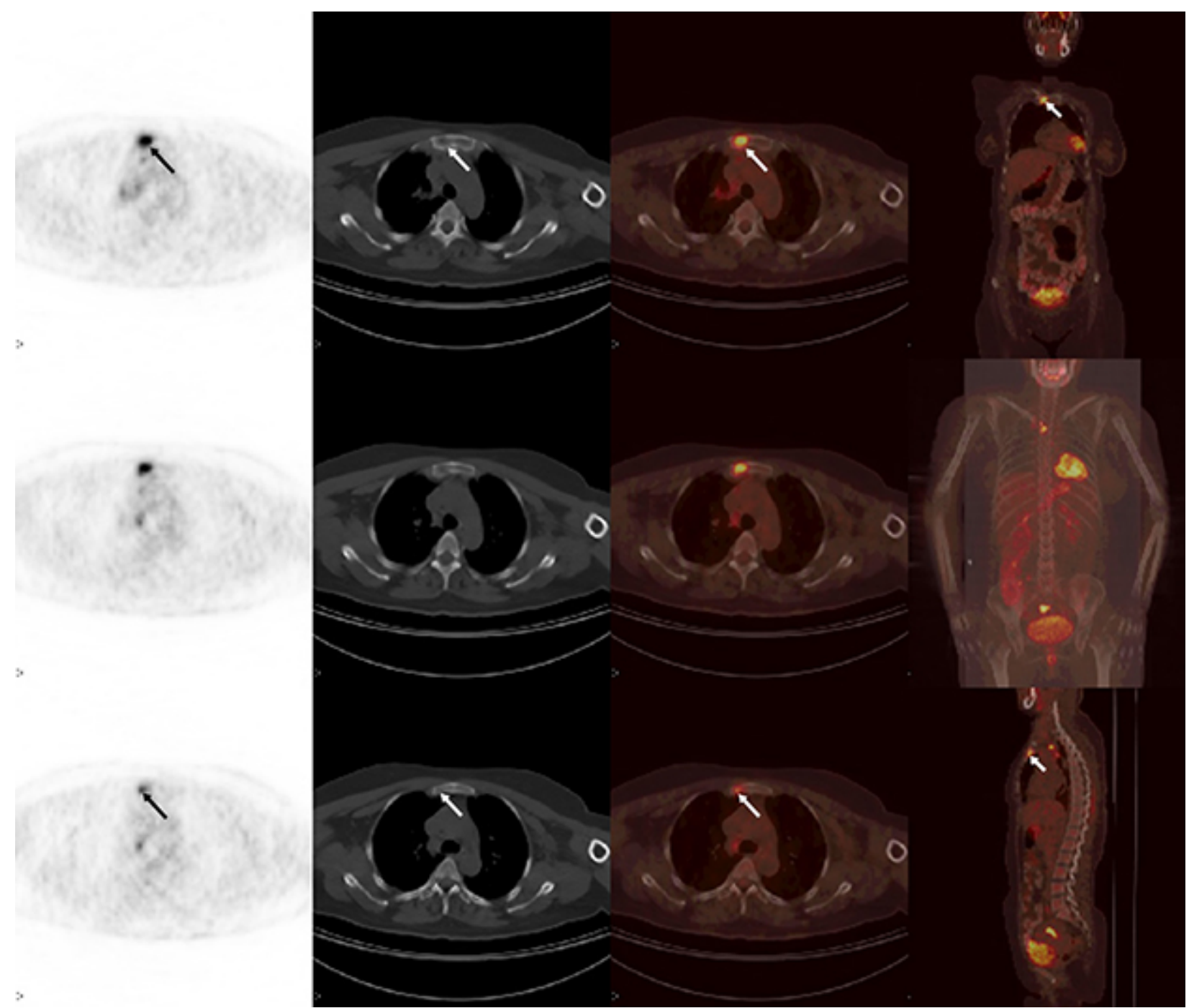

Figure 4. PET/CT images in a 51-year-old female patient. Mastectomy and axillary dissection were performed a month before, and PET/CT then was performed for initial staging before chemotherapy on the basis of clinical suspicion. The hypermetabolic lesion on the right side of the manubrium sterni, which was consistent with metastases and did not cause cortical destruction on the corresponding CT slices (1st and 2nd row) and a less hypermetabolic lesion on the right side of the proximal corpus sterni (3rd row), suggesting metastases.

liver) with locally advanced breast cancer who underwent FDG-PET for initial staging. In that study (11), the rate of detection of distant metastases was higher than that reported in the study by Cermik et al. (15), possibly due to the selected patient group with locally advanced breast cancer.

Studies investigating the contribution of FDG-PET/ $\mathrm{CT}$ in breast cancer for initial staging have become more popular in recent years $(13,17,18)$. Fuster et al. (17) conducted a prospective study in 60 patients with a primary tumor larger than $3 \mathrm{~cm}$ in diameter who underwent FDG-PET/CT for preoperative staging; distant metastases were found in $8(13.3 \%)$ of them (6 bone and bone marrow, 2 lung, and 2 liver) (17). In that study, $\mathrm{PET} / \mathrm{CT}$ detected all the distant metastases previously determined by conventional methods (17). The addition of PET/CT downstaged the disease in 7 of the 60 patients and upstaged it in 18 (10 axillary lymph node metastasis, 3 extraaxillary regional lymph node, and 5 distant metastases) of the 60 patients for whom metastasis was suspected by conventional methods; as a result, FDG PET/ CT altered the disease stage in $42 \%$ of the patients (17).

In another study including 16 patients with locally advanced breast cancer who underwent FDG-PET/CT for initial staging, increased FDG uptake was seen in all primary lesions, while the mean SUVmax of the primary 
tumor was 12.2 and the average size of the primary tumor was $3.8 \mathrm{~cm}$ (18). Seven (43.7\%) of the patients had distant metastases ( 3 contralateral supraclavicular, 2 contralateral internal mammary, 3 cervical, 2 mediastinal lymph nodes, 5 bone-bone marrow, 2 lung, and 3 liver metastases) and ultimately the stage increased in 3 patients (18). In another study, FDG PET/CT detected distant metastases in 10 (25\%) of 40 patients with breast cancer (19). The authors of another study, in which 39 patients diagnosed with stage II and III breast cancer underwent FDG-PET/CT for initial staging, found distant metastases in $4(10.2 \%)$ patients ( 3 bone and bone marrow, 1 pleura) and infraclavicular lymph node metastases in 2 patients by PET/CT (12).

In a noteworthy study including 20 patients (initial staging in 12, restaging in 8), FDG PET/CT for breast cancer staging was compared with whole-body diffusionweighted imaging (DWI). A patient based assessment of this study has shown that the diagnostic performance of FDG-PET/CT in terms of sensitivity, specificity, accuracy, positive predictive value, and negative predictive value was higher or at least equal to those of DWI. This superiority was remarkably observed with M1 lymph nodes (contralateral supraclavicular-axillary-internal mammary lymph node, $100 \%$ vs. $0 \%$ ) and bone metastases (100\% vs. $8 \%$ ). Consequently, compared with FDG-PET/ CT, DWI could not detect a higher number of lesions, except for one primary lesion in the breast. On the other hand, FDG PET/CT detected bone metastasis in 1 additional patient and pleural metastasis in 1 more patient. Considering the lesion-based analysis in the same study, it was demonstrated that the whole body DWI alone may not be appropriate for staging and restaging of breast cancer patients (20).

In a study of 41 patients with inflammatory breast cancer who underwent FDG-PET/CT for initial staging, distant metastases were detected in $20(49 \%)$ patients (9 bone and bone marrow metastases, 4 lung, 10 mediastinal lymph nodes, 6 liver, 7 other organ metastases, and 7 contralateral supraclavicular-internal mammaryaxillary lymph node metastases). In addition, ipsilateral supraclavicular lymph node metastases in 3 patients and ipsilateral internal mammary lymph node metastases in 2 patients have been reported (21). In another similar study of inflammatory breast cancer, distant metastases were observed in 18 (31\%) of 59 patients. These patients had a worse prognosis than the patients without distant metastases (22).

A recent study, with a higher number of patients compared with other studies, investigated the value of FDG PET/CT in the detection of distant metastases in the initial staging of breast cancer. Among 225 patients, 78 (35\%) were found to have distant metastases (bone/bone marrow metastases in 55, lung in 14, mediastinal lymph node in 24 , and liver metastasis in 20 ). In that study, false- positive findings were reported in 13 patients (bone/bone marrow in 7 patients, ovary in 3 , lung in 2 , mediastinal lymph node in 1, and liver in 1) and the specificity of PET/ CT was $91.2 \%$. In the same study, false-negative findings were reported in 2 patients (one of them had a lesion detected by both bone scan and MRI, but it showed no progression over a 26-month follow-up, and the other had a lesion seen by CT, and verified by biopsy, and the progression of the lesion was observed 11 months later) and the sensitivity of PET/CT was $97.4 \%$ (13).

In our study, distant metastases were seen in 76 (29.9\%) of the 254 patients with breast cancer who underwent FDG PET/CT for initial staging. This result is comparable with the PET/CT studies mentioned above. In terms of distant metastasis distribution, bone/bone marrow metastases were the most common distant metastases (55 of the 254 patients, $21.7 \%$ ). Similarly, in a study by Niikura et al. (2011), with the largest number of patients in the literature, this outcome was reported as $24.4 \%$ (13). Bone marrow micrometastases (30.6\% of all patients and $26.4 \%$ of patients without axillary metastasis) can be frequently seen in newly diagnosed breast cancers (23). Although $\mathrm{PET}$ is generally considered to be superior to CT and bone scintigraphy in detecting lytic or mixed bone metastases and bone marrow metastases, a multimodality approach is recommended for the investigation of bone metastases due to the low sensitivity of PET in detecting sclerotic bone metastases in some cases (24). In addition, sclerotic lesions without FDG accumulation can be distinguished on CT portions of PET/CT imaging by taking into consideration the appearances of lesions on corresponding CT slices (25).

When we look at the involvement of distant organs other than the bone/bone marrow in our study, 18 (7.1\%) patients had lung metastases, 33 (13\%) patients had mediastinal lymph node metastases, $12(4.8 \%)$ patients had liver metastases, 25 patients (9.8\%) had other organ/ system metastases, and $15(6 \%)$ patients had contralateral axillary-supraclavicular-internal mammary lymph node metastases (contralateral axillary in 5 cases, contralateral supraclavicular in 5 cases, and contralateral axillary and contralateral supraclavicular in 5 cases). Mittal et al. (18) found contralateral supraclavicular lymph node metastases in 3 of 16 patients, and contralateral internal mammary lymph node metastases in 2 of them, while Fuster et al. (17) observed contralateral axillary lymph node metastases in 1 of 60 patients. In addition, conglomerate hypermetabolic LAP, which suggested involvement of second primary lymphoma in the abdomen, was observed in 1 patient without extraaxillary regional lymph node metastases or/and distant metastases. It was defined as follicular lymphoma as a result of a histopathological examination. In another patient without extraaxillary regional lymph node metastases and/or distant metastases, an intense 
hypermetabolic lesion suggested second primary tumor, which was observed in the sigmoid colon. Histopathologic examination defined a high-grade tubulovillous adenoma. In a study by Weir et al. (16) a second primary tumor in the lung was detected in 1 of 84 patients.

In our series, according to the $\mathrm{T}$ stage, the rates of distant metastases in patients were as follows: $9(13.6 \%)$ of the $66 \mathrm{~T} 1$ stage patients, $46(35.7 \%)$ of the $129 \mathrm{~T} 2$ stage patients, $8(40 \%)$ of the $20 \mathrm{~T} 3$ stage patients, and $13(33.3 \%)$ of the $39 \mathrm{~T} 4$ stage patients. In addition, the frequency of metastases increased as the $\mathrm{T}$ stage increased $(\mathrm{P}=0.015)$. Distant metastases were seen at the highest rate in stage II and stage III. This result was significantly higher than that of a study conducted by Heusner et al. (19) with a limited number of patients. In contrast, the rate of detecting distant metastases was reported as $43.7 \%$ in a study including a limited number of patients with locally advanced breast cancer (stage II and above) (18). In our study, however, the incidence of metastases in stage T4 was lower than that of metastases in T2 and T3. This is probably due to the fact that inflammatory breast cancer is considered to be T4 stage regardless of tumor diameter and/or due to the unbalanced distribution of the number of patients among the groups.

In our retrospective study, distant metastases were found in 64 (41.6\%) of 154 patients (Group 1) with known breast cancer diagnosed by tru-cut/core or FNA biopsy. When we compared the SUVmax value of the primary lesions and the presence or absence of distant metastases on FDG-PET/CT images in 64 patients with distant metastases and 90 patients without distant metastases, the mean SUVmax value of the primary lesion in patients with distant metastases was $10.9 \pm 5.6(2.1-26.8)$, while it was $8.7 \pm 5.6(2.5-25.2)$ in patients without distant metastases. The difference was significant $(\mathrm{P}=0.022)$. Basu et al. (26) studied 174 patients with breast cancer, 18 (10.3\%) of whom had distant metastases, who underwent FDG-PET/ CT for initial staging and found significant correlation between the SUVmax value of the primary lesions and the detection of distant metastases. Moreover, they reported that the mean values of SUVmax of the primary lesions were $2.9 \pm 2.7$ in the group without axillary and/or distant metastasis, $4.8 \pm 3.9$ in the group with axillary metastases, and $7.7 \pm 6.2$ in the group with distant metastases. In that study, different degrees of FDG uptake in the primary lesion were observed in all 174 patients as in our Group 4 patients (26).

We detected ipsilateral parasternal lymph node metastases in $32(12.6 \%)$ of the 254 patients and ipsilateral suprainfraclavicular lymph node metastases in 44 (17.3\%) patients. Additionally, a false-positive increase in FDG uptake was observed in 1 patient that was initially suggested to be ipsilateral supraclavicular lymph node metastasis, but after histopathological examination, it was considered as an inflammatory lesion. In a study by Bellon et al. (27) including 28 patients with locally advanced breast cancer, internal mammary lymph node metastases were reported in $7(25 \%)$ of them. In our research, internal mammary lymph node metastases were detected in $1(1.5 \%)$ of 66 patients with T1 stage, $12(9.3 \%)$ of 129 patients with T2 stage, $2(10 \%)$ of 20 patients with T3 stage, and 13 (33.3\%) of 39 patients with T4 stage. Moreover, suprainfraclavicular lymph nodes were observed in $5(7.5 \%)$ of 66 patients with T1 stage, 21 (16.2\%) of 129 patients with T2 stage, $6(30 \%)$ of 20 patients with T3 stage, and $12(30.7 \%)$ of 39 patients with T4 stage.

In our study, false-positive FDG uptakes were observed in only 2 patients (in one patient there were mediastinal lymphadenopathies with intense FDG uptake, and in the other patient a nodular lesion with increased FDG uptake at the upper lobe of the right lung and a mass lesion with intense FDG uptake at the left inguinal fossa were found) that were suggested to be distant metastases, but after histopathological examinations these foci were considered inflammatory processes. Our false-positive findings are much lower than those of other similar studies in the literature. This is probably due to the evaluation of CT appearances of lesions in addition to PET findings when interpreting the PET/CT scan and not considering the physiological variants as lesions. There was a false-negative finding in only one case in FDG-PET/CT imaging. In this case, there were numerous millimeter-sized nodular lesions that were observed on CT slices of bilateral lung parenchyma and not visualized in PET images probably due to the resolution limit. Progression was observed in the clinical follow up.

One of the limitations of the present retrospective study is that histopathologic confirmation of all lesions could not be provided. A similar problem can be observed in other studies in the literature. Another limitation is that bone scintigraphy could not be performed in all patients who had bone metastases. Moreover, the body parts that could be imaged by PET/CT were not examined by CT-MR, thus eliminating the possibility of comparison between them.

In conclusion, FDG PET/CT detected metastases, especially in patients who were considered at the early stage of breast cancer. FDG-PET/CT led to a change in the disease stage and treatment approach in newly diagnosed breast cancer patients due to its superiority in detecting extraaxillary regional lymph node metastases and distant metastases. When considering the data of our study (despite its limitations) and of other studies concerning the role of FDG-PET/CT in the initial staging of breast cancer, FDG PET/CT is a preferable imaging method in the staging of newly diagnosed breast cancers, including early stage patients. More prospective studies with larger series on this subject as well as cost effectiveness analyses are highly recommended. 


\section{References}

1. Siegel R, Ward E, Brawley O, Jemal A. Cancer statistics, 2011: the impact of eliminating socioeconomic and racial disparities on premature cancer deaths. CA Cancer J Clin 2011; 61: 212236.

2. Ries LAG, Harkins D, Krapcho M, Mariotto A, Miller BA, Feuer EJ, Clegg L, Eisner MP, Horner MJ, Howlader $\mathrm{N}$ et al. SEER Cancer Statistics Review, 1975-2003 (based on November 2005 SEER data submission). Bethesda, MD, USA: National Cancer Institute; 2006.

3. Fisher B. Malignancies of the breast. In: Cameron RB, editors. Practical Oncology. Norwalk, CT, USA: Appleton \& Lange; 1994. pp. 417-434.

4. Hossfeld DK, Sherman CD, Love RR, Bosch FX. Manual of Clinical Oncology. 5th ed. Geneva, Switzerland: UICC; 1990. pp. 236-248.

5. Hance KW, Anderson WF, Devesa SS, Young HA, Levine $\mathrm{PH}$. Trends in inflammatory breast carcinoma incidence and survival: the surveillance, epidemiology, and end results program at the National Cancer Institute. J Natl Cancer Inst 2005; 97: 966-975.

6. Carlson RW, McCormick B. Update: NCCN breast cancer clinical practice guidelines. J Natl Compr Canc Netw 2005; 3: S7-S11.

7. Bomanji JB, Costa DC, Ell PJ. Clinical role of positron emission tomography in oncology. Lancet Oncol 2001; 2: 157-164.

8. Hustinx R, Benard F, Alavi A. Whole-body FDG-PET imaging in the management of patients with cancer. Semin Nucl Med 2002; 32: 35-46.

9. Avril N, Rosé CA, Schelling M, Dose J, Kuhn W, Bense S, Weber W, Ziegler S, Graeff H, Schwaiger M. Breast imaging with positron emission tomography and fluorine-18 fluorodeoxyglucose: use and limitations. J Clin Oncol 2000; 18: 3495-3502.

10. Quon A, Gambhir SS. FDG-PET and beyond: molecular breast cancer imaging. J Clin Oncol 2005; 23: 1664-1673.

11. Mahner S, Schirrmacher S, Brenner W, Jenicke L, Habermann CR, Avril N, Dose-Schwarz J. Comparison between positron emission tomography using 2-[fluorine-18]fluoro-2-deoxy-Dglucose, conventional imaging and computed tomography for staging of breast cancer. Ann Oncol 2008; 19: 1249-1254.

12. Groheux D, Moretti JL, Baillet G, Espie M, Giacchetti S, Hindie E, Hennequin C, Vilcoq JR, Cuvier C, Toubert ME et al. Effect of (18)F-FDG PET/CT imaging in patients with clinical Stage II and III breast cancer. Int J Radiat Oncol Biol Phys 2008; 71: 695-704.

13. Niikura N, Costelloe CM, Madewell JE, Hayashi N, Yu TK, Liu J, Palla SL, Tokuda Y, Theriault RL, Hortobagyi GN et al. FDG$\mathrm{PET} / \mathrm{CT}$ compared with conventional imaging in the detection of distant metastases of primary breast cancer. Oncologist 2011; 16: 1111-1119.
14. Schirrmeister $H$, Kühn $T$, Guhlmann A, Santjohanser C, Hörster T, Nüssle K, Koretz K, Glatting G, Rieber A, Kreienberg R et al. Fluorine-18 2-deoxy-2-fluoro-D-glucose PET in the preoperative staging of breast cancer: comparison with the standard staging procedures. Eur J Nucl Med 2001; 28: 351-358.

15. Cermik TF, Mavi A, Basu S, Alavi A. Impact of FDG PET on the preoperative staging of newly diagnosed breast cancer. Eur J Nucl Med Mol I 2008; 35: 475-483.

16. Weir L, Worsley D, Bernstein V. The value of FDG positron emission tomography in the management of patients with breast cancer. Breast J 2005; 11: 204-209.

17. Fuster D, Duch J, Paredes P, Velasco M, Muñoz M, Santamaría G, Fontanillas M, Pons F. Preoperative staging of large primary breast cancer with $[18 \mathrm{~F}]$ fluorodeoxyglucose positron emission tomography/computed tomography compared with conventional imaging procedures. J Clin Oncol 2008; 26: 4746-4751.

18. Mittal BR, Manohar K, Kashyap R, Bhattacharya A, Singh B, Singh G. The role of (18)F-FDG PET/CT in initial staging of patients with locally advanced breast carcinoma with an emphasis on M staging. Hell J Nucl Med 2011; 14: 135-139.

19. Heusner TA, Kuemmel S, Umutlu L, Koeninger A, Freudenberg LS, Hauth EA, Kimmig KR, Forsting M, Bockisch A, Antoch G. Breast cancer staging in a single session: whole-body PET/CT mammography. J Nucl Med 2008; 49: 1215-1222.

20. Heusner TA, Kuemmel S, Koeninger A, Hamami ME, Hahn S, Quinsten A, Bockisch A, Forsting M, Lauenstein T, Antoch $G$ et al. Diagnostic value of diffusion-weighted magnetic resonance imaging (DWI) compared to FDG PET/CT for whole-body breast cancer staging. Eur J Nucl Med Mol I 2010; 37: 1077-1086.

21. Carkaci S, Macapinlac HA, Cristofanilli M, Mawlawi O, Rohren E, Gonzalez Angulo AM, Dawood S, Resetkova E, Le-Petross HT, Yang WT. Retrospective study of 18F-FDG PET/CT in the diagnosis of inflammatory breast cancer: preliminary data. J Nucl Med 2009; 50: 231-238.

22. Alberini JL, Lerebours F, Wartski M, Fourme E, Le Stanc E, Gontier E, Madar O, Cherel P, Pecking AP. $18 \mathrm{~F}$-fluorodeoxyglucose positron emission tomography/ computed tomography (FDG-PET/CT) imaging in the staging and prognosis of inflammatory breast cancer. Cancer 2009; 115: 5038-5047.

23. Braun S, Vogl FD, Naume B, Janni W, Osborne MP, Coombes RC, Schlimok G, Diel IJ, Gerber B, Gebauer G et al. A pooled analysis of bone marrow micrometastasis in breast cancer. $\mathrm{N}$ Engl J Med 2005; 353: 793-802.

24. Nakai T, Okuyama C, Kubota T, Yamada K, Ushijima Y, Taniike K, Suzuki T, Nishimura T. Pitfalls of FDG-PET for the diagnosis of osteoblastic bone metastases in patients with breast cancer. Eur J Nucl Med Mol I 2005; 32: 1253-1258. 
25. Lardinois D, Weder W, Hany TF, Kamel EM, Korom S, Seifert B, von Schulthess GK, Steinert HC. Staging of non-small-cell lung cancer with integrated positron-emission tomography and computed tomography. N Engl J Med 2003; 348: 25002507.

26. Basu S, Mavi A, Cermik T, Houseni M, Alavi A. Implications of standardized uptake value measurements of the primary lesions in proven cases of breast carcinoma with different degree of disease burden at diagnosis: does 2-deoxy-2-[F-18] fluoro-D-glucose-positron emission tomography predict tumor biology? Mol Imaging Biol 2008; 10: 62-66.
27. Bellon JR, Livingston RB, Eubank WB, Gralow JR, Ellis GK, Dunnwald LK, Mankoff DA. Evaluation of the internal mammary lymph nodes by FDG-PET in locally advanced breast cancer (LABC). Am J Clin Oncol 2004; 27: 407-410. 